\title{
Usos de la Sangre de drago (Croton Lechleri Müll) en apósitos para heridas crónicas obtenidos mediante la técnica de Electrospinning
}

\author{
Gabriel Melo ${ }^{\mathrm{a}}$, Nathaly Villacís ${ }^{\mathrm{a}}$, Karla Vizuete ${ }^{\mathrm{b}}$, Carlos Arroyo ${ }^{\mathrm{b}}$, Christian Narváez ${ }^{\mathrm{a}}$ \\ ${ }^{a}$ Departamento de Ciencias de la Energía y Mecánica, Universidad de las Fuerzas Armadas ESPE \\ ${ }^{\mathrm{b}}$ Centro de Nanociencia y Nanotecnología, Universidad de las Fuerzas Armadas ESPE \\ jgmelo@espe.edu.ec, cnvillacis@espe.edu.ec, ksvizuete@espe.eu.ed, ccarroyo@ espe.edu.ec, cpnarvaez1@espe.edu.ec
}

\begin{abstract}
Resumen-El presente estudio tiene como principal objetivo dar uso a un material ancestral poco usado como es la sangre de drago (Croton Lechleri Müll), tecnificándolo por primera vez a través de un proceso tecnológico como el electrospinning para la obtención de fibras y partículas a escalas nano y micrométricas proyectándolas a posteriores aplicaciones en apósitos bioactivos para heridas crónicas, que aprovechen las propiedades curativas (antibacteriales, cicatrizantes, antibióticas, antioxidantes, etc.) de la savia y las características morfológicas de las estructuras propias de la técnica. Se consideraron varias concentraciones de sangre de drago en diferentes soluciones poliméricas base, siendo principal la solución de fibroína de seda aprovechando las propiedades de la seda en aplicaciones de ingeniería de tejidos desarrollando de 4 alternativas de apósito analizadas mediante microscopia electrónica de barrido (SEM) y finalmente aproximando cual sería la mejor para la aplicación antes definida.
\end{abstract}

Palabras Claves-Apósito, Electrospinning, Sangre de Drago.

Abstract-The goal of this study is to use an unused ancestral material such as drago's blood (Croton Lechleri Müll), using it for the first time through a technological process such as electrospinning to obtain fibers and particles at scales nano and micrometers projecting them to subsequent applications in bioactive wound dressings, which take advantage of the healing properties (antibacterial, healing, antibiotics, antioxidants, etc.) of the sap and the morphological characteristics of the structures of the technique. Several concentrations of drago blood were considered in different base polymeric solutions, the main one being the silk fibroin solution that exploits the properties of silk in tissue engineering applications of 4 analysis alternatives analyzed by scanning electron microscopy (SEM) and finally approximating which would be the best alternative for the previously defined application.

Keywords-Wound dressing, Electrospinning, Drago's blood.

\section{INTRODUCCIÓN}

Los apósitos o wound dressing son elementos que ayudan a evitar la infección y mantener un entorno apropiado que permita la correcta cicatrización de la herida [1], para ello deben contar con ciertas características esenciales como la capacidad hemostática o antihemorrágica, poder antibacteriano, absorción de excesos de líquidos exudados como fluidos de la herida o pus, transmisión apropiada entre agua y vapor, capacidad de ajustarse al contorno de la herida, adhesión funcional al tejido sano, no al herido, indoloro para el paciente y finalmente que pueda removerse con facilidad [2-5]. Lograr estas propiedades es posible con procesos tecnológicos como el electrospinning, proceso utilizado para la formación de fibras a escala nano y micrométrica. Las nanofibras electrohiladas cuentan con una serie de propiedades que las fortalecen como materiales para apósitos y vendaje de heridas tales como la facilidad de trabajar con una serie de polímeros biocompatibles, alta permeabilidad al oxígeno, tamaño de poro variable, alta relación de área superficial a volumen, pequeños diámetros de fibra que se asemejan a la matriz extracelular (EM) y al área de superficie grande de una fibra que permite unir las proteínas y los receptores de la membrana celular durante el proceso de cultivo celular. Por lo tanto, son capaces de potenciar la proliferación celular [6]-[9]. En la producción de apósitos según Chen et. al. [10], dependiendo de las necesidades del sujeto o paciente son varios los agentes dopantes aplicables a la membrana para mejorar su acción sobre la herida, por ejemplo, si lo que se busca son propiedades homeostáticas se usaría fibrogeno, para propiedades antibacteriales partículas de plata o antibióticos, para propiedades antinflamatorias caléndula o con factores de crecimiento. Curiosamente dichas propiedades son abarcadas por la sangre de drago, una savia usada ancestralmente para una serie de aplicaciones médicas.

La sangre de drago o Croton lechleri Müll es un árbol amazónico de pequeña envergadura común en las laderas andinas orientales de Perú, Colombia, Bolivia y Ecuador. Históricamente su savia se ha utilizado sobre heridas para detener la hemorragia, acelerar el proceso de cicatrización evitando dejar huellas visibles o queloideas, sellar y proteger las lesiones de la infección, secándose rápidamente y generando una barrera de tono rojizo, formando una "segunda piel" [11]. Este componente es usado principalmente para el tratamiento de una serie de patologías [12]-[16]. Su componente activo principal es un elemento llamado taspina, alcaloide encargado de la formación del colágeno y por ende la aceleración de la cicatrización, además de ser un excelente antibacteriano y antiséptico [17]-[21]. Vaisberg et al. [22] demostraron que la aplicación tópica diaria de $0,05 \mathrm{ml}$ de una solución al 10\% de la savia a heridas cutáneas presentadas por ratones causa un aumento significativo del $31 \%$ en la tasa de reparación de la herida, corroborando que el efecto de cicatrización está estrechamente relacionado con la existencia de taspina y la convierte en un material biológicamente adecuado para aplicaciones de curación de heridas.

En el siguiente artículo se describen algunas alternativas de apósitos para heridas crónicas en la piel de una solución 
polimérica base con sangre de drago obtenida mediante la técnica de electrospinning. En la sección II se presenta los materiales y métodos utilizados en la experimentación, en la sección III se presenta el análisis de resultados y finalmente, las conclusiones relevantes son presentadas en la sección IV.

\section{MATERIALES Y MÉTODOS}

\section{A. Materiales y Equipos}

Producto del proceso de experimentación y dado que no existen registros de haber usado la savia previamente en electrospinning se usó Sangre de drago orgánica certificada de la Fundación Chankuap en Macas, Ecuador, capullos de seda Bombyx Mori de la empresa Bao Jian Zhen, quitosán sintetizado Chemsavers de pureza 99\%, Óxido de polietileno (PEO) de 1000KDa S3 Chemical, Polyvinil alcohol (PVA) de 15KDa LobaChemie, Polyvinil pirrolodona (PVP) de 30KDa LobaChemie y Ácido acético glacial de pureza del 99\% del laboratorio J.T. Baker. Además, para la preparación de muestras se usó un agitador magnético Thermo Scientific S130815y una balanza analítica Shimadzu TW423L de apreciación 0.01g.

\section{B. Protocolo de preparación de muestras}

Para mezclar la sangre de drago con una solución polimérica base se utilizó la fórmula de concentración volumen/volumen descrita en (1):

$$
X \%_{v / v}=\frac{V_{\text {soluto }}}{V_{\text {solución }}}=\frac{V_{\text {Sdrago }}}{V_{\text {Sdrago }}+V_{\text {Soluciónbase }}}
$$

Usando los instrumentos de laboratorio y el equipo de seguridad adecuados se midió el volumen necesario de solución polimérica base y sangre de drago, colocándolas en un frasco de plástico con un agitador magnético, sellándolo y posteriormente colocándolo en la plancha de agitación donde se agitó durante 10-15 minutos a velocidad constante, corroborando que el sistema de calentamiento esté apagado.

\section{Electrospinning}

Una vez preparada la solución se la lleva a la máquina de electrospinning Basic Research Y-Flow, mostrada en la Fig. 1.

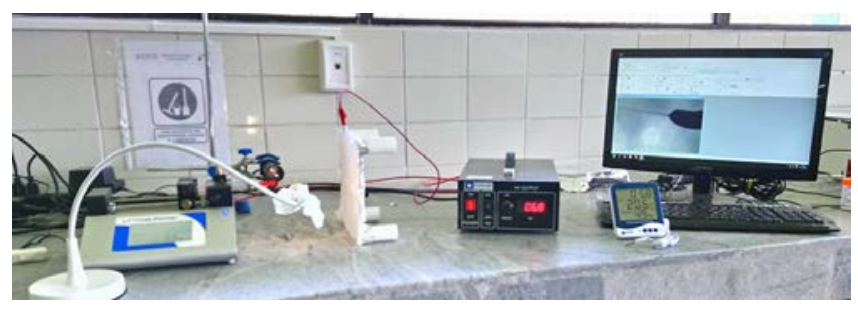

Fig. 1. Máquina de electrospinning Basic Research

La solución es colocada en una jeringa convencional de $10 \mathrm{~mL}$, posteriormente se la ubica en la bomba de infusión, se conecta tanto el polo negativo al capilar como el positivo al colector previamente preparado y se prende la bomba y la fuente de alto voltaje procurando en la variación de la tensión genere el campo eléctrico necesario para romper la tensión superficial de la solución y generar el cono de Taylor visible en la pantalla que al estabilizarse corrobora la formación de fibras o partículas dependiendo de la solución polimérica base.

\section{ANÁLISIS DE RESULTADOS}

Se mezcló sangre de drago con una serie de polímeros sintéticos y biopolímeros teniendo inconvenientes de solubilidad cuando está concentrada al 100\%, donde al mezclar la savia con una solución acuosa de Fibroína de Seda + PEO 3\%w/v en distintas concentraciones $100 \%$, $50 \%, 25 \%, 10 \% \mathrm{v} / \mathrm{v}$, la solución tendía a formar una mezcla altamente viscosa, no homogénea y grumosa imposible de electrohilar.

Debido a que no es posible generar una mezcla homogénea entre soluciones poliméricas y la sangre de drago, se realizó un estudio experimental de la solubilidad de la savia frente a otros polímeros y solventes. La sangre de drago concentrada o diluida en solventes como agua o etanol únicamente se mezcla con polímeros de bajo peso molecular para la generar fibras (quitosán, PEO) o partículas (PVP).

En primer lugar se realizó un estudio de flujo estacionario de la savia en un reómetro TA-Instruments Discovery HR-2 con una precizalla a velocidad de cizalla constante de $1 \mathrm{rad} / \mathrm{s}$ para procurar uniformidad en la solución y posteriormente un ensayo de flujo estacionario a velocidades de cizalla entre 0.01 y 160 [rad/s] para obtener la gráfica que permita definir el tipo de fluido, obteniendo los resultados que se muestran en la Fig. 2. La viscosidad de la sangre de drago fluctúa entre 1,493 [Pa.s] a la velocidad de precizalla y 13,61 [Pa.s] a 160 [rad/s]. Dado que no existe una relación lineal entre el esfuerzo de corte y la tasa de cizalla y que la viscosidad decrece mientras que el esfuerzo crece frente al aumento de la velocidad de cizalla, se concluye que se trata de un fluido no newtoniano pseudoplástico.

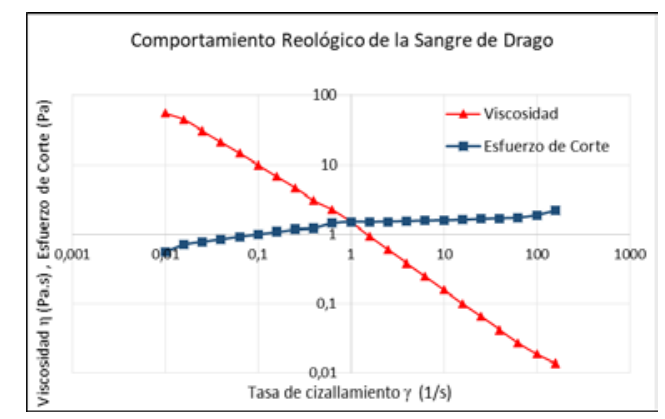

Fig. 2. Comportamiento Reológico de la sangre de drago

Todas las alternativas tienen como solución polimérica base la solución de fibroína de seda con óxido de polietileno al 3\%w/v. La fibroína de seda es un biopolímero ampliamente usado como biomaterial en el campo biomédico, especialmente en ingeniería de tejidos y en medicina regenerativa, dado que facilita la adhesión de las células, estimula su crecimiento y permite la diferenciación, además, es biocompatible, resistente y biodegradable en fases controlables, lo que lo convierte en el biomaterial con las mejores características para la producción de apósitos bioactivos. [23,24].

\section{1) Membrana de fibroína de seda con sangre de drago}

Tras poder mezclar la sangre de drago diluida al $2 \% \mathrm{v} / \mathrm{v}$ en agua con la solución de fibroína de seda, esta se electrohila 
obteniendo una membrana manipulable con presencia de beads (collares) con un diámetro medio defibra de 160 [nm] y una desviación estándar relativamente alta de 47,14 [nm] como se muestra en la Fig. 3.

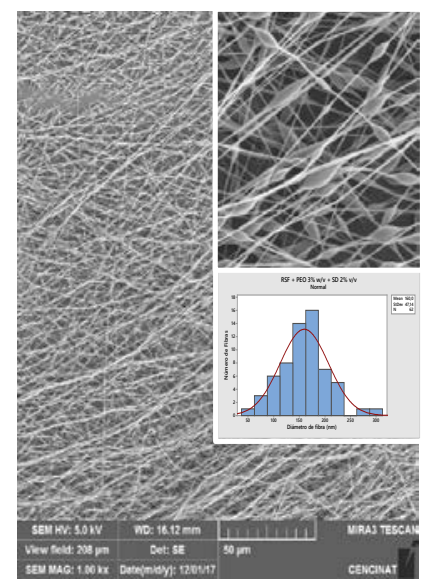

Fig. 3. Membrana de RSF + PEO $3 \% w / v$ con s. de drago al 2\%v/v

2) Nanopartículas de PVP $30 \mathrm{~K} 10 \% \mathrm{w} / \mathrm{w}+\mathrm{SD} 20 \% \mathrm{v} / \mathrm{v}$

Otra de las alternativas dado que fue posible generar partículas con una solución polimérica de PVP fue dopar las membranas obtenidas de RSF + PEO 3\% + SD 2\% v/v con nano y micropartículas de PVP $30 \mathrm{~K}+$ SD 20\% v/v obteniendo los resultados que se muestran en la Fig. 4, donde es visible la existencia tanto de las fibras como de las partículas en su estructura.

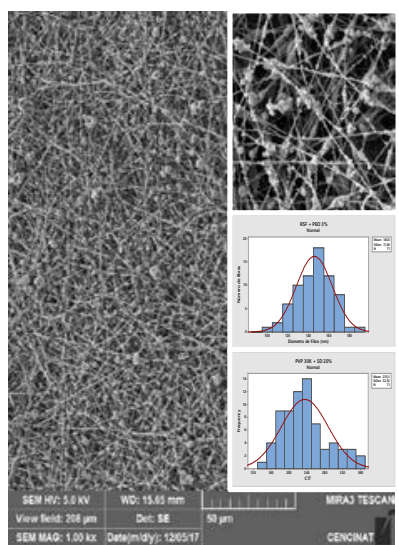

Fig. 4. Fibras de RSF-PEO3\% w/v-SD 2\%v/v dopadas con nanopartículas de PVP $30 \mathrm{~K} 13 \% \mathrm{w} / \mathrm{w}-\mathrm{SD} 20 \% \mathrm{v} / \mathrm{v}$

3) By-layer de RSF + PEO 3\%w/v + SD 2\% y CS4\% en AA $70 \%+$ PVA $10 \%+$ SD 100\%

Otra de las opciones fue realizar un by-layer uniendo una capa de RSF + PEO 3\%w/v + SD 2\% v/v con una capa de quitosán (CS) $4 \%$ en AA $70 \%$ + PVA 10\% + SD 100\% aprovechando que la solución de quitosán permitió solubilizar la sangre de drago concentrada al 100\%. De esta forma se obtuvieron los resultados mostrados en la Fig. 5, donde se observa las capas de los dos biomateriales usados. La membrana obtenida de la solución de quitosán tiene un espesor mayor dado que el radio de dibujo en el colector es mucho menor al de la fibroína. Por esta razón, a pesar de tener el mismo tiempo de deposición existe una mayor cantidad de fibras de quitosán en relación a las de fibroína de seda.

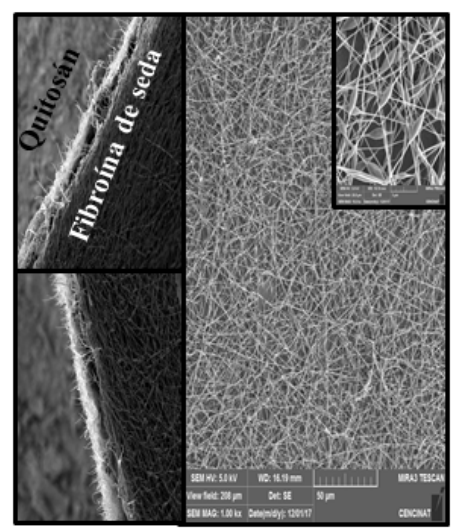

Fig. 5. By-layer de RSF + PEO 3\%w/v + SD 2\% y CS4\% en AA $70 \%+$ PVA $10 \%+$ SD $100 \%$

4) Atomización de Sangre de Drago concentrada

Una de las alternativas más sencillas es agregar directamente sangre de drago concentrada al 100\% mediante un atomizador de uso comercial, pero el inconveniente que se presenta con este método es que la fibroína de seda reconstituida o RSF obtenida de la extracción por protocolo químico al ser electrohilada es soluble al agua y dado que la sangre de drago cuenta con agua en su estructura disuelve la fibroína obteniendo la imagen del microscopio óptico que se muestra en la Fig. 6.

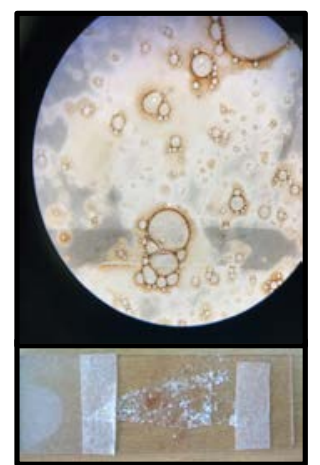

Fig. 6. Sangre de drago atomizada sobre una membrana de RSF + PEO $3 \% \mathrm{w} / \mathrm{v}$

\section{CONCLUSIONES}

La solubilidad de la sangre de drago presentó problemas a la hora de mezclarla con una serie de soluciones poliméricas, por tal motivo, se experimentó la solubilidad con ciertos polímeros sintéticos y biopolímeros; permitiendo una mezcla uniforme en polímeros de bajo peso molecular como el PVA de 15[KDa] o como el PVP 30[KDa] y generando una solución grumosa y poco uniforme con polímeros como el PEO de 1000[KDa]; esto dio lugar a que la única concentración de sangre de drago que se mezcló uniformemente con la solución de fibroína de seda sea al $2 \% \mathrm{v} / \mathrm{v}$ en agua, concentración relativamente baja frente al uso común que está concentrado al $100 \%$ y relativamente alta frente a la solución diluida que se usa en úlceras gástricas que está aproximadamente en $0.06 \%$ v/v en agua. Realizadas las 4 alternativas de apósitos, se identificó que la atomización de sangre de drago sobre la membrana de fibroína de seda no es la más adecuada; las otras 3 alternativas resultan adecuadas para la producción de un apósito siempre que se procure antes ensayos biológicos que 
analicen el comportamiento de las mismas frente agentes que impiden la correcta cicatrización de la herida. El uso de nano partículas permite usar la mezcla sobre cualquier apósito pasivo para convertirlo en bioactivo, mientras que la diferencia entre el uso de una membrana y un by-layer radica principalmente en la rigidez del apósito y la concentración de sangre de drago que se puede alcanzar en la estructura final, siendo para el primer caso del $2 \% \mathrm{v} / \mathrm{v}$ mientras que para el segundo caso es posible concentrarla al $100 \%$ en la solución de quitosán. De esta forma, la solución más adecuada en base a los estudios realizados es un bylayer de fibroína de seda (material regenerativo) con quitosán (material antibacterial) y la sangre de drago tanto por las propiedades curativas como por las propiedades mecánicas y morfológicas del apósito desarrollado.

\section{AGRADECIMIENTOS}

Este trabajo fue apoyado por el proyecto de investigación PIC-16-BENS-002 del programa becas ENSAMBLE de la SENESCYT. Se agradece al Laboratorio de Reología y Fluidos Complejos y el Laboratorio de Microscopia del Centro de Nanociencia y Nanotecnología la Universidad de las Fuerzas Armadas ESPE. Del mismo modo, un agradecimiento al Dr. Salvador Aznár Cervantes por su colaboración activa en el desarrollo del estudio.

\section{REFERENCIAS}

[1] N. Percival, "Classification of wounds and their management," Surgery (Oxford), vol. 20, nº 5, pp. 114-117, 2002.

[2] J. Venugopal, L. Ma y S. Ramakrishna, "Biocompatible nanofiber matrices for engineering dermal substitute for skin regeneration," Tissue Engineering, 2005.

[3] S. R. Bhattarai, N. Bhattarai, H. Yi, P. Hwang, D. Cha y H. Kim, "Novel biodegradable electrospun membrane: scaffold for tissue engineering,” Biomaterials. 25, pp. 2595-2602, 2004.

[4] P. Zahedi, I. Rezaeian, S. O. Ranaei-Siadat, S. H. Jafari y P. Supaphol, "A review on wound dressings with an emphasis on electrospun nanofibrous polymeric bandages," Polymers for Advanced Technologies 21, pp. 77-95, 2010.

[5] G. T. Lionelli y T. W. Lawrence, "Wound dressings," Surg. Clin. North Am. 83, p. 617, 2003.

[6] L. A. Smith y P. X. Ma, "Nano-fibrous scaffolds for tissue engineering," Colloids and Surfaces B: Biointerfaces, vol. 39, pp. 125-131, 2004

[7] Y. Zhou, D. Yang, X. Chen, Q. Xu, F. Lu y J. Nie, "Electrospun water-soluble carboxyethyl chitosan/poly(vinyl alcohol) nanofibrous membrane as potential wound dressing for skin regeneration," Biomacromolecules, vol. 9, pp. 349-354, 2006.

[8] M. S. Khil, D. Cha, H. Kim, I. Kim y N. Bhattari, «Electrospun nanofibrous polyurethane membrane as wound dressing,» Biomed. Res. 67B, pp. 675-679, 2003.

[9] X. Xu, J. Zhang y Y. Fan, Biomacromolecules, pp. 2283-2289, 2010.

[10] S. Chen, L. Bing, M. Carlson, A. Gombart, D. Reilly y J. Xie, "Recent advances in electrospun nanofibers for wound healing Review,” Nanomedicine, pp. 107-115, 2017.

[11] D. Gupta, B. Bleakley y R. K. Gupta, "Dragon’s blood: Botany, chemistry and therapeutic uses," Journal of Ethnopharmacology, 115, p. 361-380, 2008.

[12] T. Cerutti, "Plantas Medicinales Cultivo, importancia y formas de uso,” EsSalud - IMET Iquitos,, pp. 72-73, 2000.

[13] A. J. Marcelo, C. Calderon, D. Medina, M. Valencia, M. Pariona y M. Meza, Desarrollando Nuestra Diversidad Biocultural "Sangre de grado" y el Reto de su Producción Sustentable en el Perú, Lima: Gráficos S.R . Ltd, 1999.

[14] G. Persinos-Perdue, R. N. Blomster, D. A. Blake y N. R. Farnsworth, "South American Plants II: Taspine isolation and anti-inflammatory activity,” J. Pharm. Sci. 68, pp. 124-126, 1979.

[15] A. Domínguez, Fitoquímica y Caracterización del alcaloide, USA: Editorial WHO, 1985.

[16] Z. P. Chen, "Studies on the anti-tumour, anti-bacterial and woundhealing properties of dragon’s blood,” Planta Med, 1994.
[17] J. Lobardo, Diccionario Médico, España: Ediciones Doyna. Pág. 400, 1994.

[18] A. Herforth, "Antifungal plants of the Peruvian Amazon: A Survey of ethnomedicinal uses and biological activity," Cornell University USA: Honors Thesis, 2002.

[19] A. Vaisber y J. Millan, Taspine in the Cicatrizant Principle in Sangre de Grado Extracte from (Croton lechlerii), Córdova. Argentina: Plantas M.C Vol. 5, 1989.

[20] S. N. Elliot, A. Buret, W. McKnight, M. Miller y J. L. Wallace, "Bacteria rapidly colonize and delay the healing of gastric ulcers in rats,” Am. J Physiol Gastrointest Liver Physiol. 275, pp. 424-432, 1998.

[21] B. H. Porras-Reyes, W. H. Lewis, J. Roman, L. Simchowitz y T. A Mustoe, "Enhancement of wound healing by the alkaloid taspine defining mechanism of action,” Proc. Soc. Exp. Biol. Med. 203, pp 18-25, 1993.

[22] P. Allaica Tenesaca, Comparación del efecto cicatrizante de tinturas elaboradas a base de guarango (caesalpinia spinosa) y sangre de drago (croton lechleri) aplicados en ratones (mus musculus)., Riobamba: Tesis, 2015.

[23] M. Calafat, G. Guinea, J. Rigueiro y G. Plaza, "Usos médicos de la seda,” Investigación y Ciencia, pp. 28-35, 2011.

[24] U. Kim, J. Park, H. Kim, M. Wada y D. Kaplan, "Three dimensional aqueous-derived biomaterial scaffolds from silk fibroin," Biomaterials. 26, pp. 2775-2785, 2005 